\title{
Subcutaneous granulocyte colony-stimulating factor administration for subacute traumatic spinal cord injuries, report of neurological and functional outcomes: a double-blind randomized controlled clinical trial
}

\author{
Nazi Derakhshanrad, MD, PhD, ${ }^{1}$ Hooshang Saberi, MD, MPH, ${ }^{1,2}$ Mir Saeed Yekaninejad, PhD, ${ }^{3}$ and \\ Mohammad Taghi Joghataei, $\mathrm{PhD}^{4}$
}

${ }^{1}$ Brain and Spinal cord Injury Research Center, Neuroscience Institute, and 2Department of Neurosurgery, Imam Khomeini Hospital, Tehran University of Medical Sciences; ${ }^{3}$ Department of Epidemiology and Biostatistics, School of Public Health, Tehran University of Medical Sciences; and ${ }^{4}$ Neuroscience Department, School of Advanced Technologies in Medicine, Iran University of Medical Sciences, Tehran, Iran

OBJECTIVE Granulocyte-colony stimulating factor (G-CSF) is a major cytokine that has already been clinically verified for chronic traumatic spinal cord injuries (TSCls). In this study, the authors set out to determine the safety and efficacy of G-CSF administration for neurological and functional improvement in subacute, incomplete TSCl.

METHODS This phase II/III, prospective, double-blind, placebo-controlled, parallel randomized clinical trial was performed in 60 eligible patients ( 30 treatment, 30 placebo). Patients with incomplete subacute TSCls with American Spinal Injury Association Impairment Scale (AIS) grades B, C, and D were enrolled. Patients were assessed using the International Standards for Neurological Classification of Spinal Cord Injury (ISNCSCI) scale, Spinal Cord Independence Measure (SCIM-III) and International Association of Neurorestoratology Spinal Cord Injury Functional Rating Scale (IANR-SCIFRS), just before intervention and at 1,3 , and 6 months, after 7 daily subcutaneous administrations of $300 \mu \mathrm{g} /$ day of G-CSF in the treatment group and placebo in the control group.

RESULTS Among 60 participants, 28 patients $(93.3 \%)$ in the G-CSF group and 26 patients $(86.6 \%)$ in the placebo group completed the study protocol. After 6 months of follow-up, the AIS grade remained unchanged in the placebo group, while in the G-CSF group 5 patients (45.5\%) improved from AIS grade B to C, $5(45.5 \%)$ improved from AIS grade $C$ to grade $D$, and 1 patient (16.7\%) improved from AIS grade D to E. The mean \pm SEM change in ISNCSCI motor score in the G-CSF group was $14.9 \pm 2.6$ points, which was significantly greater than in the placebo group (1.4 \pm 0.34 points, $p<0.001)$. The mean \pm SEM light-touch and pinprick sensory scores improved by $8.8 \pm 1.9$ and $10.7 \pm 2.6$ points in the G-CSF group, while those in the placebo group improved by $2.5 \pm 0.60$ and $1.2 \pm 0.40$ points, $(p=0.005$ and 0.002 , respectively). Evaluation of functional improvement according to the IANR-SCIFRS instrument revealed significantly more functional improvement in the G-CSF group $(10.3 \pm 1.3$ points than in the placebo group $(3.0 \pm 0.81$ points; $p<0.001)$. A significant difference was also observed between the 2 groups as measured by the SCIM-III instrument (29.6 \pm 4.1 vs $10.3 \pm 2.2, p<0.001)$.

CONCLUSIONS Incomplete subacute TSCI is associated with significant motor, sensory, and functional improvement after administration of G-CSF.

Clinical trial registration no.: IRCT201407177441N3 (www.irct.ir)

https://thejns.org/doi/abs/10.3171/2018.6.SPINE18209

KEYWORDS spinal cord injury; granulocyte colony-stimulating factor; neurological improvement; clinical trial

ABBREVIATIONS AIS = ASIA Impairment Scale; ASIA = American Spinal Injury Association; BASIR = Brain and Spinal cord Injury Research; CONSORT = Consolidated Standards of Reporting Trials; CBC = complete blood count; G-CSF = granulocyte colony-stimulating factor; IANR-SCIFRS = International Association of Neurorestoratology Spinal Cord Injury Functional Rating Scale; ISNCSCI = International Standards for Neurological Classification of Spinal Cord Injury; MAS = Modified Ashworth Scale; MPSS = methylprednisolone sodium succinate; SCIM-III = Spinal Cord Independence Measure version III; TSCI = traumatic spinal cord injury; TUMS = Tehran University of Medical Sciences; VAS = visual analog scale; WBC $=$ white blood cell.

SUBMITTED February 21, 2018. ACCEPTED June 7, 2018.

INCLUDE WHEN CITING Published online October 12, 2018; DOI: 10.3171/2018.6.SPINE18209. 
$\mathrm{T}$ Raumatic spinal cord injury (TSCI) produces devastating physical, social, and vocational impairment. ${ }^{2}$ Despite the availability of advanced rehabilitation modalities, there remains a need to explore therapeutic strategies for treating TSCI patients by cellular and/or molecular mechanisms modifying the pathophysiology of TSCI. New therapeutic approaches involve strategies aimed at blocking multiple mechanisms of progressive pathogenesis of secondary TSCI. ${ }^{37}$ The primary injury event starts a pathobiological cascade of secondary mechanisms that unfold in different phases, beginning within seconds of the primary trauma, and may continue for several weeks thereafter. ${ }^{38}$

Secondary injury may be an appropriate target for neuroprotective and clinical therapeutic interventions, ${ }^{12,37}$ such as methylprednisolone sodium succinate (MPSS) and the related compound tirilazad mesylate, ${ }^{4,9}$ nimodipine, ${ }^{24}$ naloxone, ${ }^{10}$ gancyclidine ( $N$-methyl-D-aspartate antagonist) ${ }^{32}$ and granulocyte colony-stimulating factor (G-CSF). ${ }^{6,14}$, ${ }^{27,33} \mathrm{G}-\mathrm{CSF}$ has already been reported to have positive effects on the course of various neurological disorders experimentally, such as stroke, ${ }^{30}$ Parkinson's disease,${ }^{20} \mathrm{Alz}$ heimer's disease, ${ }^{34}$ amyotrophic lateral sclerosis, ${ }^{23}$ sciatic nerve injury, ${ }^{17}$ partial cochlear nerve lesion, ${ }^{21}$ traumatic brain injury, ${ }^{31}$ and various rodent models of TSCI. ${ }^{22}$

Multiple preclinical studies in TSCI models have suggested several mechanisms for the neurorestorative effects of G-CSF, including antiapoptotic activity, stem cell mobilization, angiogenesis, neurogenesis, and immunomodulation through specific signaling pathways. ${ }^{18}$ Various authors have reported on G-CSF regarding its wide neuroprotective action and its neurodegenerative mechanisms of action in the treatment of TSCI.$^{18} \mathrm{G}$-CSF has been successfully used clinically for acute ${ }^{14,33}$ and chronic ${ }^{6,27}$ TSCI, as well as subacute compressive myelopathy ${ }^{28,29}$ and stroke in humans. ${ }^{25}$

Previously, on the basis of the available preclinical and clinical results described, we have conducted phase $\mathrm{I},{ }^{6}$ phase I/IIa, ${ }^{27}$ and phase $\mathrm{III}^{7}$ clinical trials with promising results regarding safety, feasibility, and efficacy of GCSF as a neuroprotective therapy in patients with chronic TSCI. In this trial, a phase II/III study, we attempt to determine the safety and verify the efficacy of subcutaneous G-CSF in comparison with placebo, for the treatment of subacute incomplete TSCI and compare neurological as well as functional outcomes between the 2 groups.

\section{Methods \\ Study Design}

This phase II/III, prospective, double-blind, placebocontrolled, parallel randomized clinical trial, was conducted from August 2014 to June 2017 in the Brain and Spinal cord Injury Research (BASIR) Center at Imam Khomeini Hospital, Tehran University of Medical Sciences (TUMS). The local institutional review board/ethics committee of TUMS approved the trial. In addition, the study was registered in the Iranian Registry of Clinical Trials (IRCT; registration no. IRCT201407177441N3, www.irct.ir) before enrollment of the participants. The study protocol adheres to the criteria of the revised Consolidated Standards of Re- porting Trials' (CONSORT) 2010 statement. The study was conducted in compliance with the seventh revision of the Declaration of Helsinki and the international conference on harmonization of Good Clinical Practice guidelines.

The patients were selected among outpatient TSCI cases in the BASIR Center at Imam Hospital, TUMS. Sixty eligible patients with subacute incomplete TSCI were chosen and enrolled after providing written informed consent for the study. At the screening visits, demographic and clinical assessments were performed by obtaining the patient's history, performing a physical examination, and reviewing the images.

Baseline evaluations included duration of injury, neurological level, severity of injury according to the American Spinal Injury Association (ASIA) Impairment Scale (AIS) grade (A-E), surgical approach, and concurrent complications. Neurological complications were evaluated as follows: neuropathic pain using the visual analog scale (VAS; score range 0-100) and spasticity using the Modified Ashworth Scale (MAS; score range 0-4). Neurological changes were assessed using the International Standards for Neurological Classification of Spinal Cord Injury (ISNCSCI) scale designed by ASIA (motor scores range $0-100$, light touch scores range $0-112$, and pinprick scores range $0-112 .{ }^{19}$ Functional changes were assessed using the the Spinal Cord Independence Measure III (SCIM-III; score range $0-100)^{15}$ as well as the International Association of Neurorestoratology Spinal Cord Injury Functional Rating Scale (IANR-SCIFRS; score range $0-4),{ }^{13}$ to evaluate the ability to perform basic daily tasks.

\section{Study Participants}

At the screening visits, the demographic and clinical assessments and the severity of TSCI based on AIS grading were recorded as baseline measurements. Participants between 18 and 60 years of age were enrolled in the study. Patients with incomplete (AIS grade B-D) TSCIs of between 1 and 6 months' duration (subacute phase) were selected. All patients had undergone decompression and stabilization, if necessary, in the acute setting. They also had been evaluated with postoperative CT scanning to ensure proper screw placement and MRI to ensure adequacy of the surgical decompression of the spinal canal at the same center or at our center if necessary. Neurological examination was performed, and those with upper motor neuron-type injury (Babinski sign and hyperreflexia) without evidence of lower motor neuron involvement (absence of significant muscle atrophy) were selected. Electrodiagnostic examination was performed to rule out disuse atrophy, if necessary. All patients stated that they were available to undergo the entire length of follow-up. Patients were excluded if they had active major complications associated with the TSCI or systemic associated illness.

Patients were selected among those referred to the outpatient rehabilitation clinic of BASIR Center. They were chosen during the 1.5-year recruitment period; 342 patients of the 402 patients were excluded in accordance with the CONSORT flow diagram (Fig. 1). Three hundred thirty patients did not meet inclusion criteria for the following reasons: duration of TSCI more than 6 months (chronic disease; $n=214$ ), complete SCI injury (AIS grade 


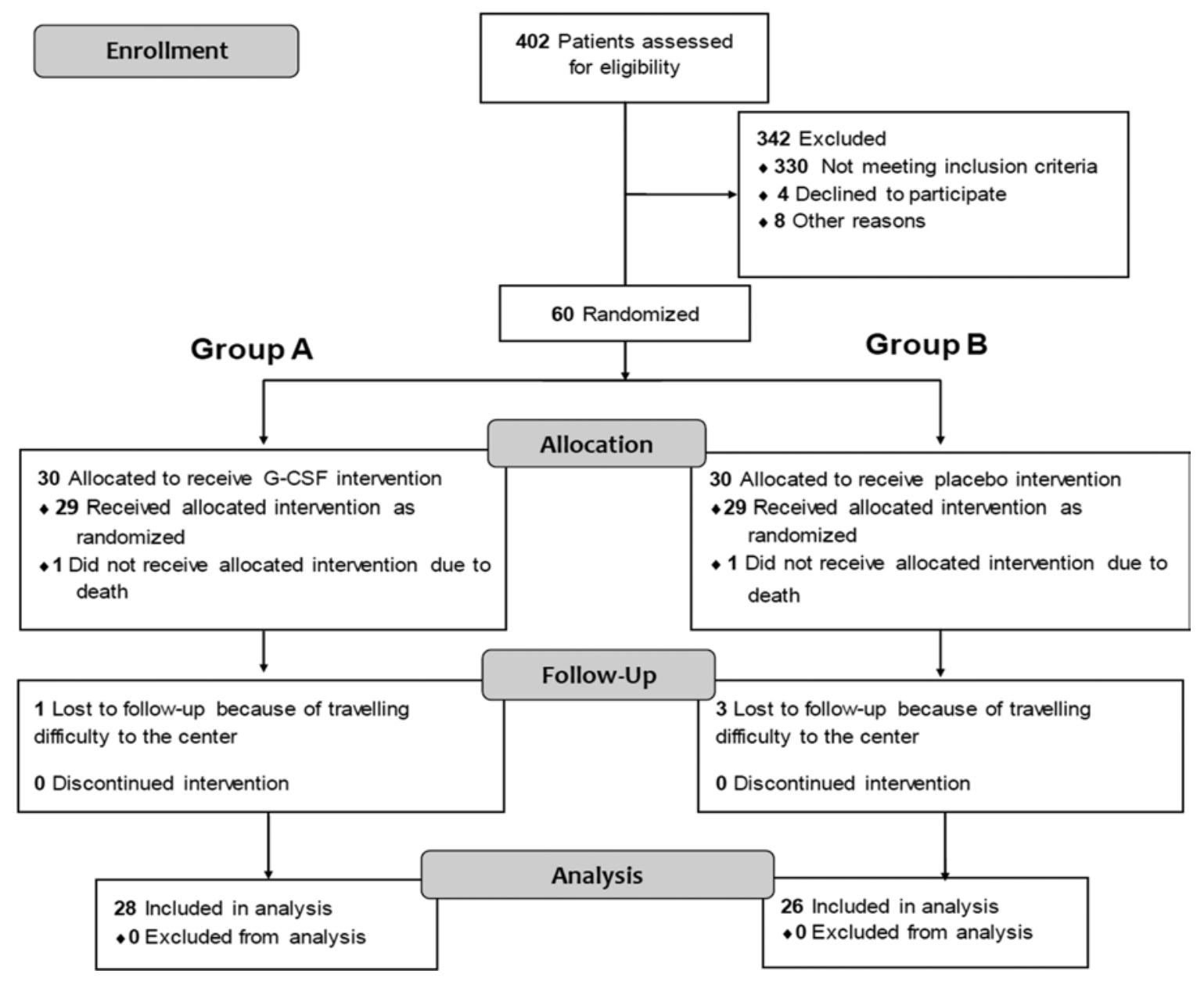

FIG. 1. CONSORT flow diagram for trial profile.

$A ; n=92)$, did not meet age requirements $(n=7)$, associated brain injury (contusion and/or diffuse axonal injury; $\mathrm{n}=2$ ), fracture of the humerus bone that led to radial nerve injury and wrist drop $(\mathrm{n}=1)$, heterotopic ossification $(n=3)$, complicated untreated grade 4 pressure sores $(n=2)$, pneumonia $(n=2)$, deep vein thrombosis $(n=4)$, metallic implant failure $(\mathrm{n}=2)$, and platelet abnormality $(n=1)$. Finally, 72 patients were found to be eligible, of whom 4 patients declined to participate and 8 patients had traveling difficulties to the center because of high cervical spinal cord injury or prolonged travel times. In total, 60 patients with TSCI were included (30 in the treatment group and 30 in the control group). They were randomly allocated into the study groups according to the study protocol as follows.

\section{Study Protocol}

After obtaining informed consent, a complete blood count (CBC) sample was obtained, and patients underwent thorough neurological assessment using the ISNCSCI sensorimotor scale. ${ }^{19}$ Functional assessment was performed using the SCIM-III ${ }^{15}$ and IANR-SCIFRS. ${ }^{13}$ Random allocation was performed using a computer-generated list obtained by an investigator with no clinical involvement in the trial. Patients were then randomized according to random block design and were allocated to the study groups using the sealed-envelope method. This was performed by an independent staff nurse who assigned the participants to the allocated interventions. Participants were primarily evaluated neurologically by an experienced neurosurgeon. Functional evaluations were performed by a trained physician. Patients and clinical evaluators were blinded with regard to the treatment groups, i.e., G-CSF and placebo vials had the same cover shape but different codes. In the treatment group, the injected vial contained $300 \mu \mathrm{g}$ G-CSF (filgrastim, Neupogen, Amgen) that was administered subcutaneously around the umbilicus with at least 2 inches of distance daily for 7 consecutive days by experienced nursing staff on an outpatient basis. In the control group the injected vial contained $1 \mathrm{ml}$ of normal saline $0.9 \%$, and the same treatment protocol was adhered to. All of these personnel were independent and unaware (blinded) of the treatment group assignment.

CBC with differential white blood cell (WBC) and platelet counts were performed at regular intervals (daily) 2-3 hours after injection, up to 7 days. The blood test was performed again at 3- and 6-month intervals after treatment to assess any chronic hematological side effects. For 
ethics concerns, if the 3 first $\mathrm{CBC}$ examination findings were normal, the test was not repeated. The control group had $3 \mathrm{CBC}$ tests, but the main researchers were unaware of their WBC count details. Possible side effects of intervention were scrutinized by an independent nursing staff and recorded. At the 1-, 3-, and 6-month follow-up visits, the patients were assessed for functional and neurological status, as well as possible secondary complications, including spasticity and neuropathic pain. In all cases, there was a single examiner for neurological assessment and another for the functional assessment from first to last follow-up visits; therefore, it was not necessary to perform an interrater reliability analysis.

All patients underwent a uniform simultaneous outpatient rehabilitation program consisting of a multidisciplinary education program that was begun during the baseline visit, with follow-ups at 1,3, and 6 months, combined with twice-weekly physical therapy together with occupational therapy as a rehabilitation package for 6 months after intervention. For handling any missing data, the last-observation-carried-forward method was used. This method replaces every missing value with the last observed value from the same patient. There was no deviation from the protocol.

\section{Primary and Secondary Outcome Measures}

Our primary outcome measure was neurological change based on ISNCSCI scores, after subcutaneous GCSF administration under investigation at a dose of 300 $\mu \mathrm{g} /$ day for 7 consecutive days, compared with a placebo, for subacute incomplete TSCIs. Motor and sensory score changes measured using the ISNCSCI scale ${ }^{19}$ from before treatment and in the follow-up visits were considered as the primary outcome measure. Functional changes, as evaluated by SCIM-III ${ }^{15}$ and IANR-SCIFRS ${ }^{13}$ before treatment and during follow-up visits were considered as secondary outcome measures. Safety was also evaluated as an important outcome measure. Any evidence of side effects was recorded regularly and reported by the independent observers during the 1st year after intervention.

Stopping guidelines for treatment included a WBC count $>50,000 / \mu l$ on $\mathrm{CBC}$, any sign of hypersensitivity reactions and/or significant thrombocytopenia (platelet count $<100,000 / \mu l$ of blood). Both were indications for discontinuation of the injections.

Sixty patients were randomized and allocated to the 2 groups (30 patients each). In the treatment group, one patient with AIS grade C cervical TSCI did not receive allocated intervention and died of respiratory failure due to Klebsiella pneumonia leading to septicemia, and another patient with AIS grade $\mathrm{C}$ was lost to follow-up because of new travel problems. In the control group, one patient with AIS grade C thoracic TSCI did not receive allocated intervention and died following a complicated gastrostomy. In addition, 3 patients ( 2 with AIS grade $\mathrm{C}$ and 1 with AIS grade D) were lost to follow-up due to new travel problems. None of the cases lost to follow-up reported any side effects on regular phone calls. Therefore, we had $10 \%$ attrition rate between the randomization and last follow-up; $54(90 \%)$ patients completed the follow-up period. There were no missing data for these patients. Analysis included
28 (11 AIS grade B, 11 AIS grade C, and 6 AIS grade D) patients in the G-CSF group and 26 (11 AIS grade B, 9 AIS grade C, and 6 AIS D) patients in the placebo group.

\section{Statistical Analysis}

Descriptive results are presented as the mean \pm SD for continuous variables and frequency with percentage for categorical variables. Statistical comparison of qualitative variables between the 2 groups was performed using the chi-square or Fisher's exact test, where appropriate. Quantitative variables in the G-CSF and placebo groups were compared using the independent sample t-test or MannWhitney test, as appropriate. The extent of AIS grade improvement, before treatment and at the 6-month follow-up, was compared between the 2 groups using the chi-square test.

The mean \pm SEM for outcome measures, including ISNCSCI motor, light touch, and pinprick scores; SCIMIII scores; and IANR-SCIFRS scores, were calculated, and the changes were reported. ANCOVA was used for comparing the change in outcome measure in the 2 groups with treatment group as the fixed effect, and baseline score as the covariate; $\mathrm{p}<0.05$ was considered as statistically significant. The sample size was calculated to detect 5 -point differences in the SCIM-III mean change ${ }^{1}$ between the 2 groups, with $85 \%$ statistical power and $5 \%$ type 1 error. Thirty patients were estimated for each group.

\section{Results}

\section{Patient Characteristics}

From August 2014 up to February 2016, 60 patients (mean age \pm SD $34.0 \pm 12.6$ years) at a single center were randomized into 2 groups: 30 patients to the G-CSF group and 30 patients to the placebo group. The overall male/ female ratio was 9:1. The follow-up period was 1 year after recruitment for each case. Twenty-eight patients (93.3\%) in the G-CSF group and 26 patients $(86.6 \%)$ in the placebo group completed the study protocol (Fig. 1).

The mean age of the participants was $36.5 \pm 13.3$ in the G-CSF group and $31.0 \pm 9.9$ years in the placebo group. There were $89.3 \%$ males in the G-CSF group and $92.3 \%$ males in the placebo group. There were no significant differences in demographic and clinical characteristics between the study groups (Table 1). Comparison of AIS grades did not show a significant difference between cervical cases $(p=0.118)$ and thoracolumbar cases $(p=0.412)$.

\section{Neurological Assessment}

There were no statistically significant differences in the baseline ISNCSCI motor and sensory scores between the G-CSF and placebo groups (Fig. 2). After 6 months of follow-up, the mean \pm SEM change in ISNCSCI motor score in the G-CSF group was $14.9 \pm 2.6$, which was significantly higher than that in the placebo group $(1.4 \pm 0.34)$ $(\mathrm{p}<0.001)$. Also, there was significant improvement in mean \pm SEM light-touch score $(8.8 \pm 1.9$ vs $2.5 \pm 0.60, \mathrm{p}=$ $0.005)$ and pinprick sensory score $(10.7 \pm 2.6$ vs $1.2 \pm 0.40$, $\mathrm{p}=0.002)$ in the G-CSF and placebo groups, respectively.

Regarding AIS grade, 5 patients $(45.5 \%)$ with AIS grade B improved to AIS grade C, while 6 patients (54.5\%) 
TABLE 1. Demographic and clinical characteristics of patients in the G-CSF and placebo groups

\begin{tabular}{|c|c|c|c|}
\hline & G-CSF Group $(n=28)$ & Placebo Group $(n=26)$ & p Value \\
\hline Mean age at time of injection, yrs & $36.5(13.3)$ & $31.0(9.9)$ & 0.096 \\
\hline Male sex & $25(89.3 \%)$ & $24(92.3 \%)$ & $>0.90$ \\
\hline Mean duration after $\mathrm{SCl}$, mos & $3.7(1.97)$ & $3.9(1.77)$ & 0.754 \\
\hline Mean education, yrs & $9.7(5.6)$ & $10.9(4.4)$ & 0.416 \\
\hline \multicolumn{3}{|l|}{ Marital status } & \multirow{3}{*}{0.847} \\
\hline Single & $9(32.1 \%)$ & $9(34.6 \%)$ & \\
\hline Married & $19(67.9 \%)$ & $17(65.4 \%)$ & \\
\hline \multicolumn{3}{|l|}{ Occupation status } & \multirow{4}{*}{0.340} \\
\hline Employed & $6(21.4 \%)$ & $5(19.2 \%)$ & \\
\hline Left job (temporary)/fired & $18(64.3 \%)$ & $13(50.0 \%)$ & \\
\hline Retired & $4(14.3 \%)$ & $8(30.8 \%)$ & \\
\hline \multicolumn{3}{|l|}{ Etiology of $\mathrm{SCl}$} & \multirow{6}{*}{0.718} \\
\hline MVA & $14(50.0 \%)$ & $14(53.8 \%)$ & \\
\hline Fall & $10(35.7 \%)$ & $8(30.8 \%)$ & \\
\hline Sport & $1(3.6 \%)$ & $2(7.7 \%)$ & \\
\hline Violence & $1(3.6 \%)$ & $2(7.7 \%)$ & \\
\hline Heavy drop & $2(7.1 \%)$ & $0(0.0 \%)$ & \\
\hline \multicolumn{3}{|l|}{ Neurological level of injury } & \multirow{3}{*}{0.554} \\
\hline Cervical & $13(46.4 \%)$ & $10(38.5 \%)$ & \\
\hline Thoracolumbar & $15(53.6 \%)$ & $16(61.5 \%)$ & \\
\hline \multicolumn{3}{|l|}{ AIS grade } & \multirow{4}{*}{0.939} \\
\hline B & $11(39.3 \%)$ & $11(42.3 \%)$ & \\
\hline C & $11(39.3 \%)$ & $9(34.6 \%)$ & \\
\hline $\mathrm{D}$ & $6(21.4 \%)$ & $6(23.1 \%)$ & \\
\hline Mean lesion length on T1WI, mm & $15.36(10.9)$ & $16.63(9.1)$ & 0.656 \\
\hline Median timing of surgical decompression, days & $3(1-9)$ & $2(1-8.7)$ & 0.825 \\
\hline Early surgical decompression ( $\leq 24 \mathrm{hrs})$ & $8(28.5 \%)$ & $11(42.3 \%)$ & \\
\hline Late surgical decompression (>24 hrs) & $19(67.9 \%)$ & $13(50.0 \%)$ & 0.431 \\
\hline No surgery & $1(3.6 \%)$ & $2(7.7 \%)$ & \\
\hline \multicolumn{3}{|l|}{ Surgical techniques } & \multirow{9}{*}{0.899} \\
\hline Pst laminectomy \& stabilization (pedicle screw) & $16(57.2 \%)$ & $13(50.0 \%)$ & \\
\hline Ant corpectomy \& stabilization (cage \& plate or screw) & $3(10.7 \%)$ & $5(19.2 \%)$ & \\
\hline Pst transpedicluar corpectomy \& stabilization & $0(0.0 \%)$ & $1(3.8 \%)$ & \\
\hline Combined approach (ant \& pst) & $2(7.1 \%)$ & $1(3.8 \%)$ & \\
\hline Traction \& pst stabilization & $2(7.1 \%)$ & $1(3.8 \%)$ & \\
\hline Traction \& ant stabilization & $3(10.7 \%)$ & $3(11.6 \%)$ & \\
\hline Laminectomy (for laminar fracture) & $1(3.6 \%)$ & $0(0.0 \%)$ & \\
\hline Conservative (traumatic cervical central cord syndrome) & $1(3.6 \%)$ & $2(7.7 \%)$ & \\
\hline Median no. of instrumented segments & $3(2-4)$ & $4(3-4)$ & 0.284 \\
\hline \multicolumn{3}{|l|}{ Rehabilitation status before study } & \multirow{4}{*}{0.684} \\
\hline Rehabilitation at home & $13(46.5 \%)$ & $15(57.7 \%)$ & \\
\hline Outpatient rehabilitation & $9(32.1 \%)$ & $6(23.1 \%)$ & \\
\hline No rehabilitation & $6(21.4 \%)$ & $5(19.2 \%)$ & \\
\hline Mean duration, mos & $2.6(2.1)$ & $2.0(1.8)$ & 0.304 \\
\hline \multicolumn{4}{|l|}{ Mean WBC count, $\mu \mathrm{l}$} \\
\hline Before treatment & $7511(1980)$ & $7252(1580)$ & 0.600 \\
\hline After treatment & $28,453(9806)$ & 8138 (4393) & $<0.001$ \\
\hline
\end{tabular}

Ant = anterior; $\mathrm{MVA}=$ motor vehicle accident; $\mathrm{pst}=$ posterior; $\mathrm{T} 1 \mathrm{WI}=\mathrm{T} 1$ - weighted imaging .

Values are presented as the number of patients (\%) unless stated otherwise. Mean values are presented as the mean (SD). Median values are presented as the median (IQR). 

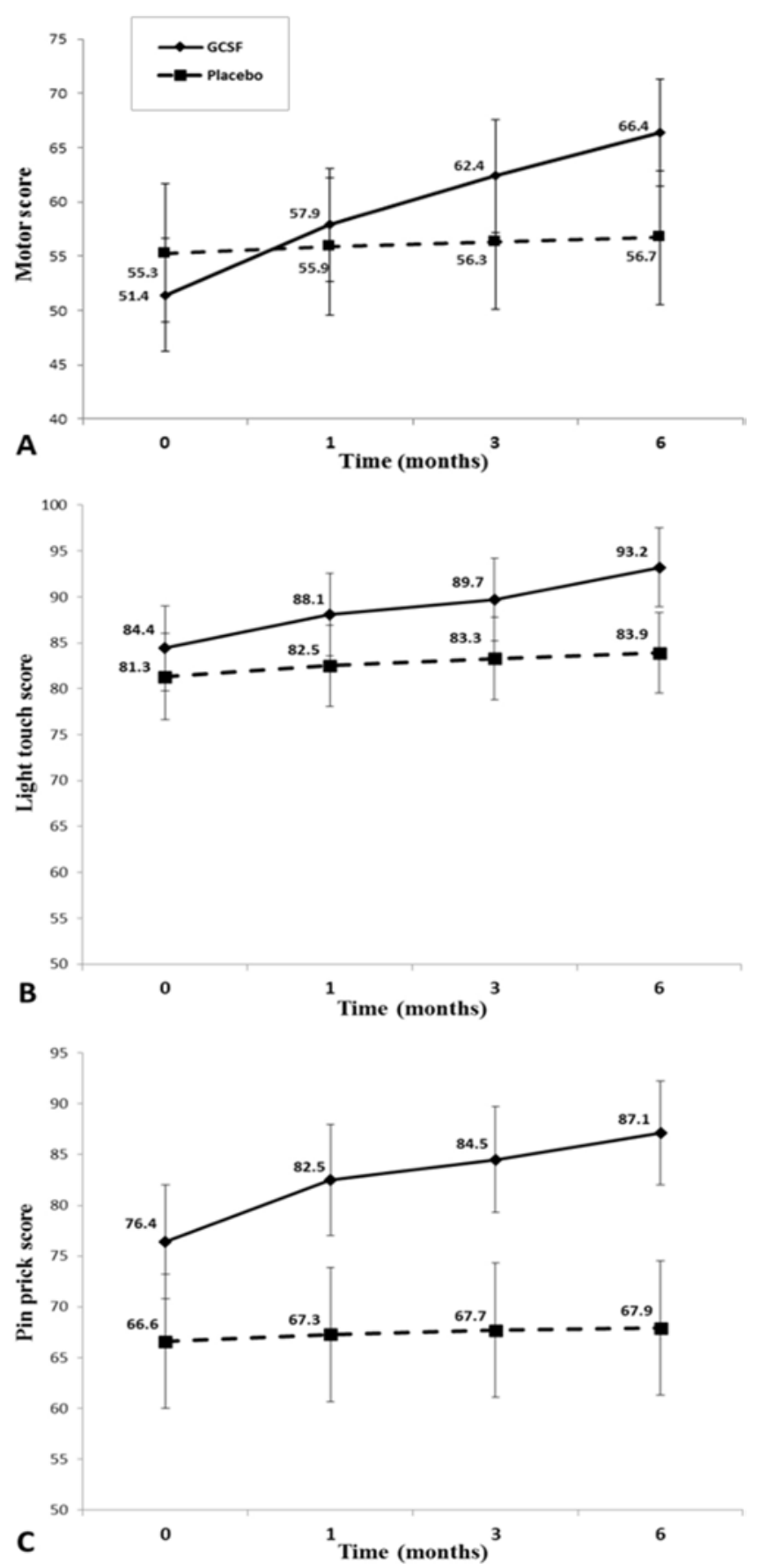

FIG. 2. Comparison of ISNCSCI scores. A: ISNCSCI motor scores. There was no significant difference between the groups before treatment $(p=0.645)$; statistical significance was observed 6 months after treatment $(p<0.001)$. B: ISNCSCI light-touch scores. There was no significant difference between the groups before treatment $(p=0.651)$; statistical significance was observed 6 months after treatment $(p=0.002)$. C: ISNCSCI pinprick scores of the 2 groups. There was no significant difference between the groups before treatment $(p=0.267)$; statistical significance was observed 6 months after treatment $(p<0.001)$. Values are means with SEM denoted by the error bars. remained unchanged. In AIS grade $\mathrm{C}$ patients, 5 patients (45.5\%) improved to AIS grade D, and 6 patients $(54.5 \%)$ were unchanged. In AIS grade D patients, 1 patient $(16.7 \%)$ improved to AIS grade E, and 5 patients (83.3\%) remained unchanged (Fig. 3A). There were no changes in AIS grade in the placebo group. Overall, $11(39.3 \%)$ of the 28 patients in the G-CSF group had a 1-grade improvement in their AIS grade compared with no AIS changes in the placebo group, a difference that was statistically significant ( $\mathrm{p}$ $<0.001)$. In the G-CSF group, the AIS grade improved in $38.4 \%$ of patients with cervical injuries and in $40 \%$ of those with thoracolumbar injuries. We found that the duration of injury had a significant effect on motor score changes $(p<0.001)$ but not on sensory changes $(p>0.2)$.

\section{Functional Assessment}

Evaluation of functional outcomes using the IANRSCIFRS instrument revealed significant changes in the G-CSF group (10.3 \pm 1.3 points), in comparison with the placebo group $(3.0 \pm 0.81$ points, $\mathrm{p}<0.001)$. In addition, assessment using the SCIM-III instrument showed a significant difference in functional changes between the 2 groups $(29.6 \pm 4.1$ vs $10.3 \pm 2.2$ points, $\mathrm{p}<0.001)$. No statistically significant difference was observed in the baseline measurements of the total value of SCIM-III and IANR-SCIFRS scores between the G-CSF and placebo groups (Fig. 3B and C). The mean progressive functional assessments are shown in Fig. 4. Regarding SCIM-III (Fig. 4A) and IANR-SCIFRS (Fig. 4B) subscale changes, patients in the G-CSF group had significant improvement in comparison to those in the placebo group.

\section{Subgroup Analysis}

A subgroup analysis for cervical and thoracolumbar regions was performed between the 2 groups (intergroup analysis). The analysis showed significant differences among all neurological and functional changes, in both cervical and thoracolumbar subgroups, between the G$\mathrm{CSF}$ and placebo cases, with the exception of the lighttouch score change in cervical subgroup $(\mathrm{p}=0.07$, near to significant; Table 2). Intragroup analysis of G-CSF cases showed greater changes in motor $(p=0.037)$ and pinprick (borderline significant, $\mathrm{p}=0.051$ ) scores in the cervical subgroup than in the thoracolumbar subgroup (Table 3). Also intragroup analysis for early and late surgical decompression in the G-CSF group showed nonsignificant changes in motor, pinprick, SCIM-III, and FRS scores. Changes in light-touch scores, however, were significant in the early decompression subgroup $(\mathrm{p}=0.012$, Table 3$)$.

\section{White Blood Cell Count}

The CBC was performed before, as well as daily after, each injection. The mean \pm SD postinjection WBC count in G-CSF group was 28,453 \pm 9806/ $\mu$ l and $8136 \pm 4393$ $\mu l$ in the placebo group. There was a significant increase in WBC in the G-CSF group compared with the placebo group $(\mathrm{p}<0.001)$.

\section{Systemic Side Effects}

In the G-CSF group, there were 2 (7.1\%) individuals 

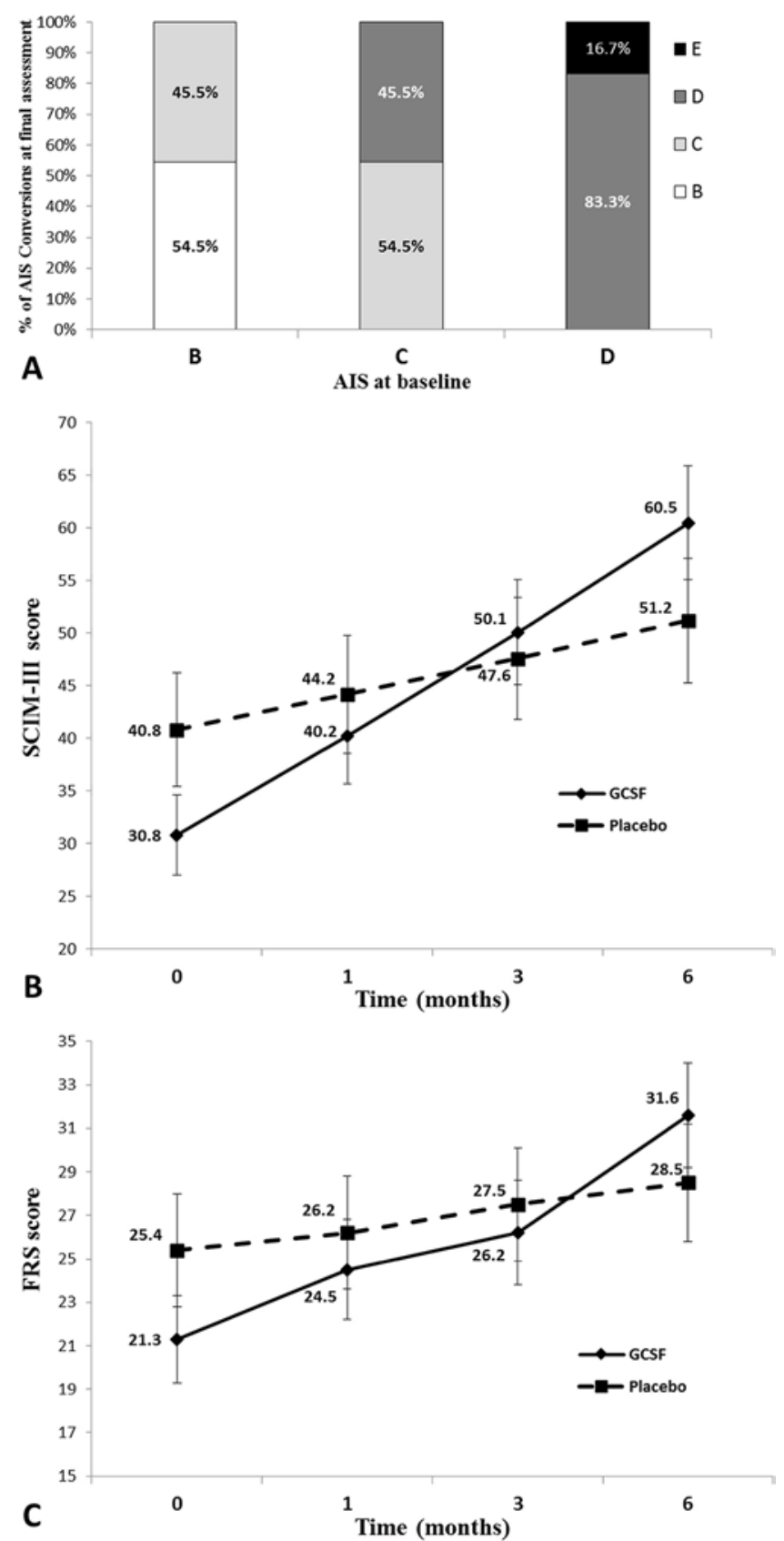

FIG. 3. A: Percentage of AIS changes at final assessment in the G-CSF group. There were no significant changes in AIS grade in the placebo group. B: Comparison of the SCIM scores. No significant difference was noted before treatment $(p=0.136)$; statistical significance was observed 6 months after treatment $(p<0.001)$. C: Comparison of the IANRSCIFRS (FRS) scores. No significant difference before treatment $(p=$ 0.219 ); statistical significance was observed 6 months after treatment ( $p$ $<0.001$ ). Values in $\mathrm{B}$ and $\mathrm{C}$ are means with SEM denoted by the error bars.

with mild to moderate bone pain, $2(7.1 \%)$ cases of transient pruritus and skin rash, 1 (3.5\%) fever, and $1(3.5 \%)$ patient with left upper quadrant pain, without splenomegaly on ultrasonography. All systemic complications subsided spontaneously for at most 1 week after the last dose of G-CSF. There were no systemic side effects in the placebo group.

\section{Neuropathic Pain and Spasticity}

Three (10.7\%) patients in the G-CSF group experienced increased neuropathic pain. Two patients had 20-point and 1 patient had 10-point increases in severity of pain according to the VAS score approximately 6 months after injection. This complication lasted up to 1 year but was amenable to GABA [gamma-aminobutyric acid] receptor agonist dose adjustment. Two (7.1\%) patients showed increased spasticity. Spasticity increased, approximately 1-2 points based on the MAS, in one case after 1 month, and the other case 6 months postinjection. In the first case, the MAS score returned to the pretreatment value after 6 months. Another patient (3.5\%) showed increased frequency of spastic episodes that were controlled by conservative management. On the other hand, 6 (21.4\%) patients showed decreased neuropathic pain (20-50 points in VAS score) from 3 to 6 months after injections. Two (7.1\%) patients showed decreased spasticity; one had a 1-point decrease (based on the MAS score), and the other patient experienced a reduced number of spasticity episodes in the 6-month follow-up.

In the placebo group, $2(8.6 \%)$ patients reported new neuropathic pain. There were $2(7.6 \%)$ individuals with increased spasticity 3 to 6 months after placebo injection. In addition, $1(3.8 \%)$ patient showed decreased neuropathic pain of approximately 40 points (based on VAS).

\section{Discussion}

Conducting phase III randomized clinical trials, when possible, has always been an important step for the establishment of new therapeutic modalities. Blindness and randomizations, when plausible, establish class I evidence for the disputed treatment, which is why we carried out the current study.

\section{Prognostication}

The neurological severity of the TSCI determines a given patient's basal neurological status on admission, and consequently this is the strongest prognostic marker. ${ }^{37}$ Therefore, patients with incomplete TSCI may be the best candidates for neurorestorative treatments, provided that the delivered substance or cells are biologically safe and the route of delivery is minimally invasive. With the same rationale, we chose incomplete (AIS grade B-D) TSCIs for this study and considered subcutaneous G-CSF as a safe intervention. ${ }^{6}$ According to our results, the greatest change in AIS grade in the G-CSF group was observed among AIS grade $\mathrm{B}$ and $\mathrm{C}$ patients. However, improvement in AIS grade D was less common than in AIS grade B or C patients.

\section{Neurological Assessment}

Authors of most studies did not assess their cases with objective methods for neurological assessments, while others reported objective neurological outcomes, such as results of electrophysiological studies (electromyography, nerve conduction studies, somatosensory evoked potentials, and motor evoked potentials) ${ }^{26}$ urodynamic studies, MR myelography, ${ }^{36}$ diffusion tensor imaging, ${ }^{40}$ and serum 

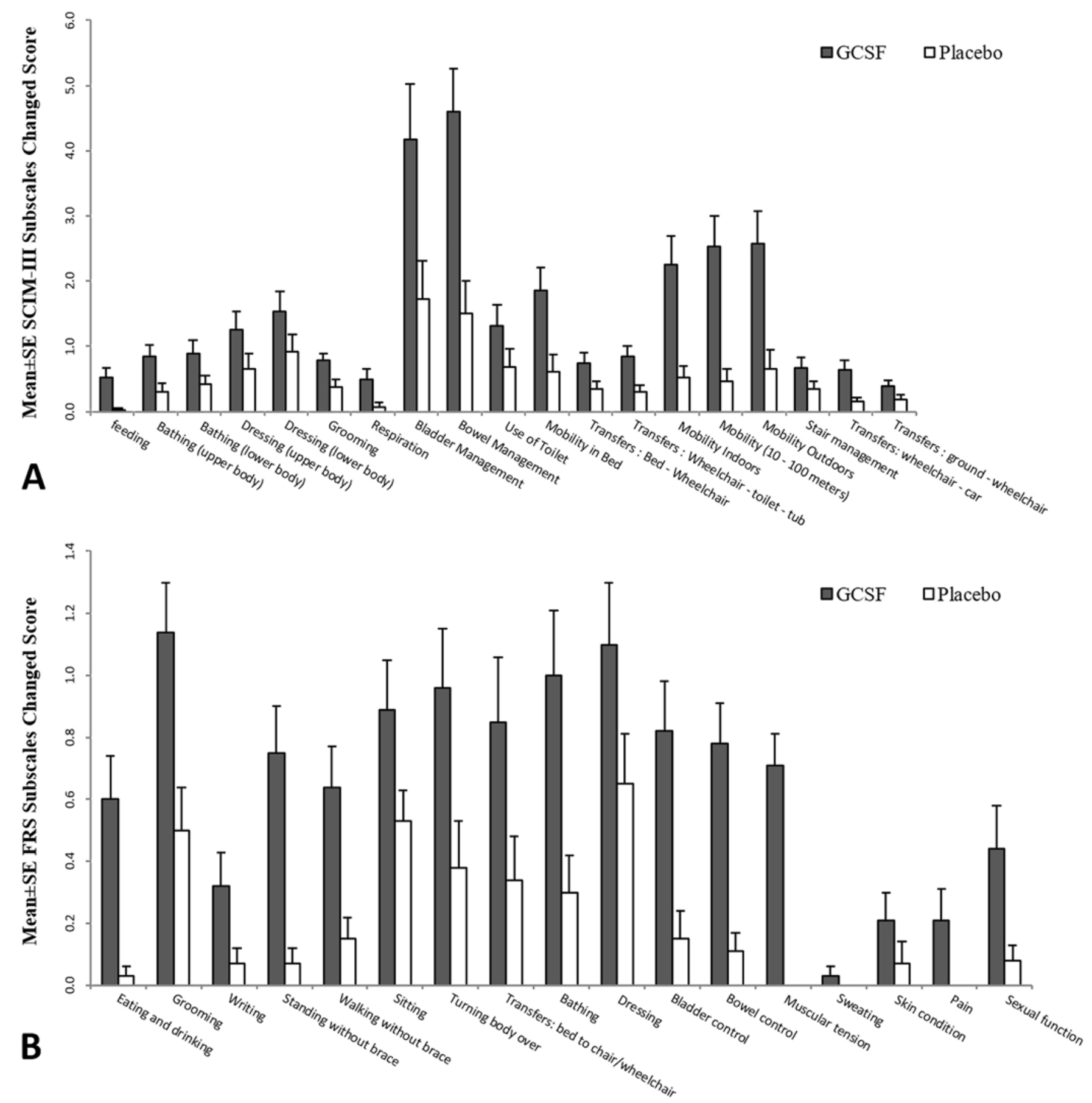

FIG. 4. Comparison of functional status at 6 months. A: SCIM score; all changes were statistically significant $(p<0.05)$ except for bathing lower body, use of toilet, bed-wheelchair transfer, and ground-wheelchair transfer. B: IANR-SCIFRS; all changes were statistically significant $(p<0.05)$ except for bathing, sweating, and skin condition.

and cerebrospinal fluid biomarkers. ${ }^{5,35}$ It seems that most authors accept ISNCSCI as the gold-standard neurological assessment, so this method was used for outcome report in our study.

\section{Timing}

The timing of neurorestorative treatments may have a great influence on outcome; it is one of the important prognosticators and sometimes a key condition for the decision to treat. Regarding the effect of duration of TSCI on outcome, we have compared our results for subacute TSCI with those from our earlier report on chronic TSCI in terms of ISNCSCI, SCIM III, and IANR-SCIFRS scores (Fig. 5). ${ }^{7}$ Better outcomes in the subacute setting may not 
TABLE 2. Subgroup analysis of change in neurological (based on ISNCSCI) and functional (based on SCIM-III and FRS) scores in G-CSF and placebo groups according to neurological level (cervical or thoracolumbar) and timing of surgical decompression (early or late)

$\begin{array}{cccc} & \text { G-CSF } & \text { Placebo } & \\ \text { Group } & \text { Group } & p \\ \text { Variable } & (n=28) & (n=26) & \text { Value }\end{array}$

Cervical level

\begin{tabular}{lccl} 
No. of patients & 13 & 10 & \\
\hline$\Delta$ Motor score & $20.8 \pm 4.0$ & $2.3 \pm 0.66$ & 0.001 \\
\hline$\Delta$ Light-touch score & $11.5 \pm 3.7$ & $3.3 \pm 1.2$ & 0.07 \\
\hline$\Delta$ Pinprick score & $16.3 \pm 5.3$ & $1.3 \pm 0.88$ & 0.02 \\
\hline$\Delta$ SCIM III score & $22.0 \pm 5.9$ & $3.2 \pm 1.2$ & 0.01 \\
\hline$\Delta$ IANR-SCIFRS score & $8.9 \pm 2.0$ & $1.4 \pm 0.87$ & 0.007 \\
\hline Thoracolumbar level & & & \\
\hline No. of patients & 15 & 16 & \\
\hline$\Delta$ Motor score & $9.8 \pm 3.0$ & $0.93 \pm 0.32$ & 0.005 \\
\hline$\Delta$ Light-touch score & $6.5 \pm 1.7$ & $2.1 \pm 0.59$ & 0.02 \\
\hline$\Delta$ Pinprick score & $5.8 \pm 1.2$ & $1.2 \pm 0.40$ & 0.001 \\
\hline$\Delta$ SCIM III score & $36.2 \pm 5.4$ & $14.8 \pm 3.1$ & 0.002 \\
\hline$\Delta$ IANR-SCIFRS score & $11.5 \pm 1.7$ & $4.0 \pm 1.1$ & 0.001 \\
\hline
\end{tabular}

Early surgical decompression $(\leq 24 \mathrm{hrs})^{*}$

\begin{tabular}{lrcc} 
No. of patients & & & \\
$\Delta$ Motor score & $19.1 \pm 4.8$ & $1.8 \pm 0.67$ & 0.001 \\
\hline$\Delta$ Light-touch score & $16.7 \pm 5.0$ & $3.0 \pm 0.76$ & 0.006 \\
\hline$\Delta$ Pinprick score & $17.8 \pm 8.7$ & $2.0 \pm 0.89$ & 0.047 \\
\hline$\Delta$ SCIM III score & $22.5 \pm 7.5$ & $10.9 \pm 3.3$ & 0.139 \\
\hline$\Delta$ IANR-SCIFRS score & $7.5 \pm 1.8$ & $3.0 \pm 1.2$ & 0.054
\end{tabular}

Late surgical decompression

$(>24 \mathrm{hrs})^{*}$

\begin{tabular}{lrcr}
\hline No. of patients & \multicolumn{1}{c}{19} & 13 & \\
\hline$\Delta$ Motor score & $13.0 \pm 3.3$ & $1.3 \pm 0.38$ & 0.007 \\
\hline$\Delta$ Light-touch score & $5.9 \pm 1.5$ & $2.3 \pm 1.0$ & 0.095 \\
\hline$\Delta$ Pinprick score & $7.8 \pm 1.4$ & $0.84 \pm 0.24$ & $<0.001$ \\
\hline$\Delta$ SCIM III score & $31.6 \pm 5.1$ & $9.6 \pm 3.6$ & 0.003 \\
\hline$\Delta$ IANR-SCIFRS score & $11.3 \pm 1.7$ & $2.8 \pm 1.2$ & 0.001 \\
\hline
\end{tabular}

$\Delta=$ change from baseline to 6 months posttreatment.

Values are presented as the mean \pm SEM unless noted otherwise.

* Surgical decompression was not performed in 1 patient in the G-CSF group and 2 patients in the placebo group.

only be due to the treatment effect size but also as a result of autorecovery mechanisms in subacute TSCI. ${ }^{8}$ Autorecovery is explicitly the cause of all neurological improvements in our control group and some of the changes in the G-CSF group. The rate of autorecovery is a function of neurological severity (AIS). ${ }^{8}$

Another important aspect of the therapeutic time window for neurorestorative intervention is that the majority of patients may arrive in the hospital several hours after the onset of injury, especially in underdeveloped healthcare settings. ${ }^{18}$ The wide therapeutic time window of G-
TABLE 3. Subgroup analysis of changes in the neurological (ISNCSCI) and functional (SCIM-III and IANR-SCIFRS) scores in the G-CSF group according to neurological level and timing of surgical decompression

\begin{tabular}{lccc}
\hline \multicolumn{1}{c}{ Variable } & $\begin{array}{c}\text { Cervical Level } \\
\text { Group }\end{array}$ & $\begin{array}{c}\text { Thoracolumbar } \\
\text { Level Group }\end{array}$ & $\begin{array}{c}p \\
\text { Value }\end{array}$ \\
\hline No. of patients & 13 & 15 & \\
\hline$\Delta$ Motor score & $20.8 \pm 4.0$ & $9.8 \pm 3.0$ & 0.037 \\
\hline$\Delta$ Light-touch score & $11.5 \pm 3.7$ & $6.5 \pm 1.7$ & 0.213 \\
\hline$\Delta$ Pinprick score & $16.3 \pm 5.3$ & $5.8 \pm 1.2$ & 0.051 \\
\hline$\Delta$ SCIM III score & $22.07 \pm 5.9$ & $36.2 \pm 4.1$ & 0.092 \\
\hline$\Delta$ IANR-SCIFRS score & $8.9 \pm 2.0$ & $11.5 \pm 1.7$ & 0.342 \\
\hline & Early Surgical & Late Surgical & \\
\multicolumn{1}{c}{ Variable* } & Group $(\leq 24$ hrs $)$ & Group $(>24$ hrs $)$ & Value \\
\hline No. of patients & 8 & 19 & \\
\hline$\Delta$ Motor score & $19.1 \pm 4.8$ & $13.0 \pm 3.3$ & 0.325 \\
\hline$\Delta$ Light-touch score & $16.7 \pm 5.0$ & $5.9 \pm 1.5$ & 0.012 \\
\hline$\Delta$ Pinprick score & $17.8 \pm 8.7$ & $7.8 \pm 1.4$ & 0.104 \\
\hline$\Delta$ SCIM III score & $22.5 \pm 7.5$ & $31.6 \pm 5.1$ & 0.338 \\
\hline$\Delta$ IANR-SCIFRS score & $7.5 \pm 1.8$ & $11.3 \pm 1.7$ & 0.215 \\
\hline
\end{tabular}

* One patient did not undergo decompression.

CSF treatement may be beneficial in unpredictable circumstances.

\section{Side Effects}

Complications and side effects of various neurorestorative treatments may be either due to the specific type of cell and/or drug applied or as result of the route of treatment delivery. The long-term reported adverse effects include accentuation of neuropathic pain, spasticity, autonomic dysreflexia, myelomalacia, and encephalomyelitis. Transient reported untoward effects include neurological deterioration, aseptic meningitis, leakage of cerebrospinal fluid, fever, headache, skin rash, and hepatic dysfunction..$^{26}$

Mild symptoms after G-CSF administration include low-back and pelvic pain, fever, chills, headache, rash, nausea, vomiting, and mild hepatic dysfunction. Symptoms have been transient and disappeared 2 to 7 days after cessation of the drug., ${ }^{3,6,14}$ In our series, patients reported transient bone pain, rash, pruritus, and left upper quadrant pain. Moreover, in our study, increased neuropathic pain (10.7\%) and spasticity (7.1\%) were among the persistent but treatable side effects. Overall, the dose (300 $\mu \mathrm{g} / \mathrm{day})$, duration (7 consecutive days), and route (subcutaneous injection) of G-CSF administration employed in the present study are generally safe and tolerable for use in subacute incomplete TSCI.

\section{Combination Therapy}

Combined neuroprotective and neuroregenerative strategies are most likely effective steps moving forward in tackling the multifaceted nature of the injury; however, this approach requires tailoring to specific patient sub- 


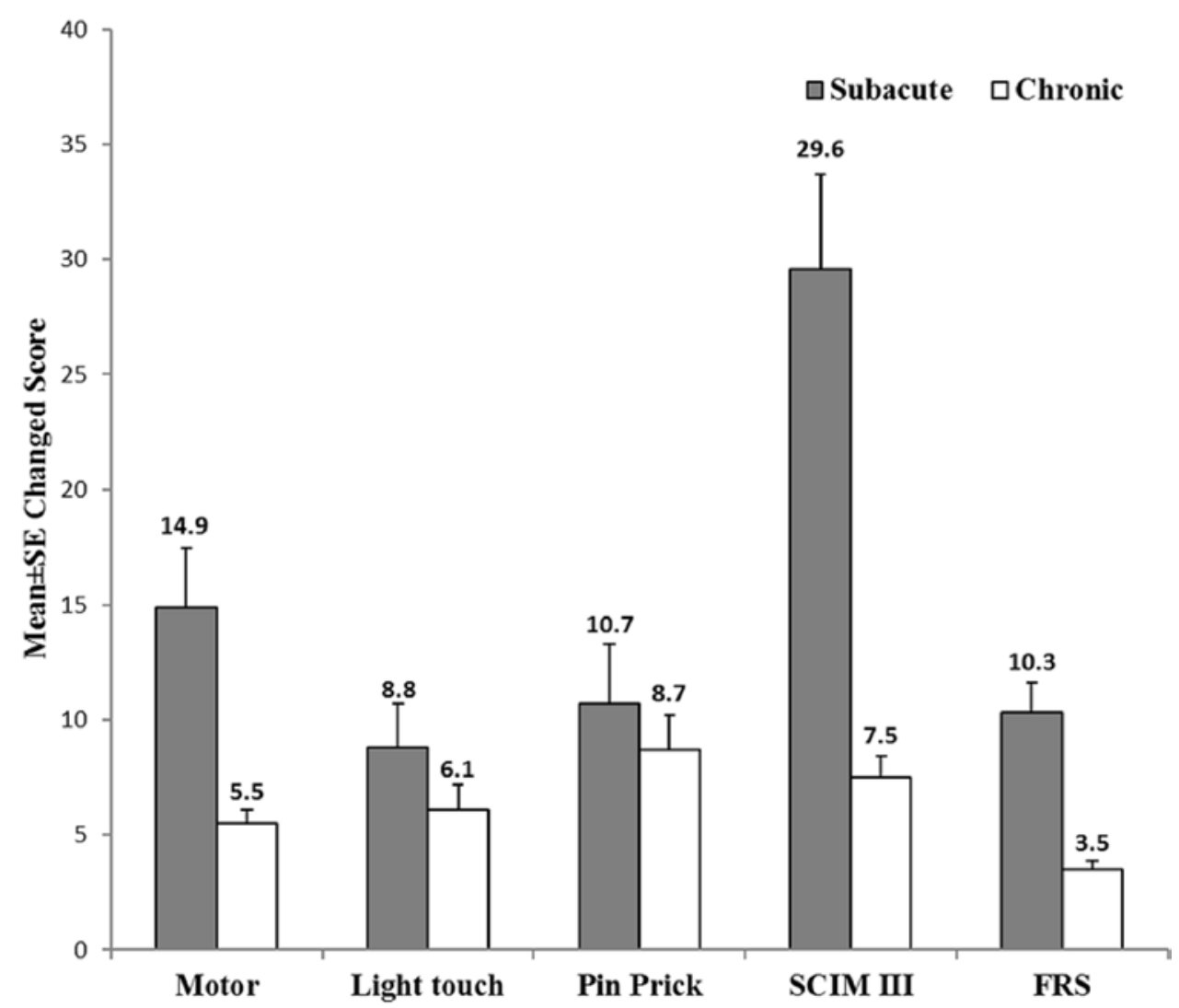

FIG. 5. Comparison of neurological (based on ISNCSCI) and functional (based on SCIM-III and FRS) score changes in subacute (this study) versus chronic (our previous study ${ }^{7}$ ) TSCls treated with G-CSF.

groups. ${ }^{2}$ MPSS commonly is administered within the first 24 hours after TSCI. ${ }^{9}$ G-CSF benefits from a much longer therapeutic time window and may be administered later in the subacute phase. ${ }^{28,29}$ Comparative studies have observed greater ISNCSCI motor score changes in a G-CSF group compared with an MPSS group. ${ }^{33}$ In addition, on 2 other studies ${ }^{16,33}$ of patients with acute TSCI, the incidence of pneumonia in the MPSS group was significantly greater than in the G-CSF group. There seems to be no contraindication for administration of the 2 drugs in the same person, i.e., they may be given to the same patient but at different times following trauma. The far superior safety and tolerability profile of G-CSF make it a suitable potential adjunct to MPSS after the acute phase of SCI treatment.

\section{Cervical and Thoracolumbar Subgroups}

Subgroup analysis of our findings (Table 3) showed a significant difference in neurological and functional outcomes between cervical and thoracolumbar cases, although both subgroups benefit from treatment. This finding is concordant with those of Wu et al. ${ }^{39}$ (acidic fibroblast growth factor), and Grossman et al. ${ }^{11}$ (riluzole). Also, better response was observed in our cervical cases in the G-CSF group, similar to the results of the Casha et al. (minocycline) study.

\section{Strengths}

With the randomized controlled design with parallel assigned, concealed allocation, and double-blindness of examiners and participants in our study, we have tried to eliminate the risks of systematic bias; selection bias; bias of patient background and baseline neurological and functional status difference (baseline imbalance) of the treatment arms; the impact of placebo effect; participant bias; and measurement bias and exclude the effect of autorecovery in the G-CSF group.

The G-CSF and placebo groups were treated at a single institution with the same method of rehabilitation, and/or time periods. Hence, treatment consistency between the 2 groups has not been compromised.

The pathophysiology and symptoms of TSCI may vary, depending on the spinal level of injury (cervical or thoracolumbar), as well as the severity of the trauma measured by the AIS. We tried to exlude this effect in our study by eliminating selection bias between the two groups. Further analysis revealed that the initial AIS grade and neurological level of injury were the same between the 2 groups due to the random block design of the study. Also, this study had minimal attrition bias (10\%), with $90 \%$ of patients having complete follow-up and minimal missing data due to regular contacts and follow-up visit appointment reminders.

Regarding cost-effectiveness of the treatment, subcutaneous G-CSF administration may be administered during hospital admission, especially in subacute cases on an 
outpatient basis, provided that the patient is cooperative and well instructed. Interobserver bias was minimal in this study because all examinations were performed by constant evaluators during the study for neurological and functional assessments.

\section{Limitations}

There is a probability of information bias due to observer errors of assessment tools, including the ISNCSCI, SCIM III, and IANR-SCIFRS. Another limitation is that 4 patients were lost to follow-up and 2 patients died before receiving allocated intervention. We tried to minimize their number with periodic phone calls for fixing the assessment meetings with the patient. Finally, we should mention that the total number of included patients was relatively small.

\section{Conclusions}

Incomplete subacute TSCI is associated with significant motor, sensory, and functional improvement due to autorecovery, which this may be significantly promoted by G-CSF administration. Further multicenter trials would be the next step for establishment of the results.

\section{Acknowledgments}

The study was funded by Tehran University of Medical Sciences Deputy of Research.

We acknowledge the TSCI patients who enrolled in this trial for their kind participation in the study protocol. Our thanks go to Miss Asal Derakhshanrad, MSc; Miss Mahnaz Niavarani, MSc; and Mr. Sadegh Bijani, BS for their kind contribution in the study. Also we appreciate Dr. Amirhossein Tavakkoli, psychiatrist; Miss Manije Yazdanshenas, MSc, psychologist; Dr. Abolghassem Nikfallah, MD, urologist; Dr. Mohsen Maghari, MD, coloproctologist; Mr. Amir Hassan Kohan, MSc, occupational therapist; and Mrs. Marjan Hassan, MSc, physiotherapist, for their great contribution to the outpatient education program.

\section{References}

1. Ackerman P, Morrison SA, McDowell S, Vazquez L: Using the Spinal Cord Independence Measure III to measure functional recovery in a post-acute spinal cord injury program. Spinal Cord 48:380-387, 2010

2. Ahuja CS, Martin AR, Fehlings M: Recent advances in managing a spinal cord injury secondary to trauma. F1000Res 5(F1000 Faculty Rev):1017, 2016

3. Anderlini P, Przepiorka D, Seong D, Miller P, Sundberg J, Lichtiger B, et al: Clinical toxicity and laboratory effects of granulocyte-colony-stimulating factor (filgrastim) mobilization and blood stem cell apheresis from normal donors, and analysis of charges for the procedures. Transfusion 36:590595,1996

4. Bracken MB, Shepard MJ, Holford TR, Leo-Summers L, Aldrich EF, Fazl M, et al: Administration of methylprednisolone for 24 or 48 hours or tirilazad mesylate for 48 hours in the treatment of acute spinal cord injury. Results of the Third National Acute Spinal Cord Injury Randomized Controlled Trial. National Acute Spinal Cord Injury Study. JAMA 277:1597-1604, 1997

5. Casha S, Zygun D, McGowan MD, Bains I, Yong VW, Hurlbert RJ: Results of a phase II placebo-controlled randomized trial of minocycline in acute spinal cord injury. Brain 135:1224-1236, 2012
6. Derakhshanrad N, Saberi H, Yekaninejad MS, Eskandari G, Mardani A, Rahdari F, et al: Safety of granulocyte colonystimulating factor (G-CSF) administration for postrehabilitated motor complete spinal cord injury patients: an open-label, phase I study. Cell Transplant 22 (Suppl 1):S139-S146, 2013

7. Derakhshanrad N, Saberi H, Yekaninejad MS, Joghataei MT, Sheikhrezaei A: Granulocyte-colony stimulating factor administration for neurological improvement in patients with postrehabilitation chronic incomplete traumatic spinal cord injuries: a double-blind randomized controlled clinical trial. J Neurosurg Spine 29:97-107, 2018

8. Fawcett JW, Curt A, Steeves JD, Coleman WP, Tuszynski $\mathrm{MH}$, Lammertse D, et al: Guidelines for the conduct of clinical trials for spinal cord injury as developed by the ICCP panel: spontaneous recovery after spinal cord injury and statistical power needed for therapeutic clinical trials. Spinal Cord 45:190-205, 2007

9. Fehlings MG, Wilson JR, Harrop JS, Kwon BK, Tetreault LA, Arnold PM, et al: Efficacy and safety of methylprednisolone sodium succinate in acute spinal cord injury: a systematic review. Global Spine J 7 (3 Suppl):116S-137S, 2017

10. Flamm ES, Young W, Collins WF, Piepmeier J, Clifton GL, Fischer B: A phase I trial of naloxone treatment in acute spinal cord injury. J Neurosurg 63:390-397, 1985

11. Grossman RG, Fehlings MG, Frankowski RF, Burau KD, Chow DS, Tator C, et al: A prospective, multicenter, phase I matched-comparison group trial of safety, pharmacokinetics, and preliminary efficacy of riluzole in patients with traumatic spinal cord injury. J Neurotrauma 31:239-255, 2014

12. Hawryluk GW, Rowland J, Kwon BK, Fehlings MG: Protection and repair of the injured spinal cord: a review of completed, ongoing, and planned clinical trials for acute spinal cord injury. Neurosurg Focus 25(5):E14, 2008

13. Huang H, Xi H, Chen L, Zhang F, Liu Y: Long-term outcome of olfactory ensheathing cell therapy for patients with complete chronic spinal cord injury. Cell Transplant 21 (Suppl 1):S23-S31, 2012

14. Inada T, Takahashi H, Yamazaki M, Okawa A, Sakuma T, Kato K, et al: Multicenter prospective nonrandomized controlled clinical trial to prove neurotherapeutic effects of granulocyte colony-stimulating factor for acute spinal cord injury: analyses of follow-up cases after at least 1 year. Spine (Phila Pa 1976) 39:213-219, 2014

15. Itzkovich M, Gelernter I, Biering-Sorensen F, Weeks C, Laramee MT, Craven BC, et al: The Spinal Cord Independence Measure (SCIM) version III: reliability and validity in a multi-center international study. Disabil Rehabil 29:19261933, 2007

16. Kamiya K, Koda M, Furuya T, Kato K, Takahashi H, Sakuma $\mathrm{T}$, et al: Neuroprotective therapy with granulocyte colonystimulating factor in acute spinal cord injury: a comparison with high-dose methylprednisolone as a historical control. Eur Spine J 24:963-967, 2015

17. Keiner D, von Pein H, Sommer C, Heimann A, Kempski O, Oertel J: Does G-CSF have a regenerative influence on traumatic lesions of the sciatic nerve in rats? J Neurosurg 119:A563, 2013 (Abstract 733)

18. Khorasanizadeh M, Eskian M, Vaccaro AR, RahimiMovaghar V: Granulocyte colony-stimulating factor (G-CSF) for the treatment of spinal cord injury. CNS Drugs 31:911937, 2017

19. Kirshblum SC, Burns SP, Biering-Sorensen F, Donovan W, Graves DE, Jha A, et al: International standards for neurological classification of spinal cord injury (revised 2011). J Spinal Cord Med 34:535-546, 2011

20. Meuer K, Pitzer C, Teismann P, Krüger C, Göricke B, Laage $\mathrm{R}$, et al: Granulocyte-colony stimulating factor is neuroprotective in a model of Parkinson's disease. J Neurochem 97:675-686, 2006 
21. Montibeller GR, Schackmann B, Urbschat S, Oertel JMK: Effect of granulocyte colony-stimulating factor on the cochlear nuclei after creation of a partial nerve lesion: an experimental study in rats. J Neurosurg 128:296-303, 2018

22. Pitzer C, Klussmann S, Krüger C, Letellier E, Plaas C, Dittgen $\mathrm{T}$, et al: The hematopoietic factor granulocyte-colony stimulating factor improves outcome in experimental spinal cord injury. J Neurochem 113:930-942, 2010

23. Pitzer C, Krüger C, Plaas C, Kirsch F, Dittgen T, Müller R, et al: Granulocyte-colony stimulating factor improves outcome in a mouse model of amyotrophic lateral sclerosis. Brain 131:3335-3347, 2008

24. Pointillart V, Petitjean ME, Wiart L, Vital JM, Lassié P, Thicoipé M, et al: Pharmacological therapy of spinal cord injury during the acute phase. Spinal Cord 38:71-76, 2000

25. Ringelstein EB, Thijs V, Norrving B, Chamorro A, Aichner $\mathrm{F}$, Grond $\mathrm{M}$, et al: Granulocyte colony-stimulating factor in patients with acute ischemic stroke: results of the AX200 for Ischemic Stroke trial. Stroke 44:2681-2687, 2013

26. Saberi H, Derakhshanrad N, Yekaninejad M: Review of recently documented clinical neuroprotective and cellular treatment for spinal cord injury: an analysis of outcomes. $\mathbf{J}$ Neurorestoratol 2:15-24, 2014

27. Saberi H, Derakhshanrad N, Yekaninejad MS: Comparison of neurological and functional outcomes after administration of granulocyte-colony-stimulating factor in motor-complete versus motor-incomplete postrehabilitated, chronic spinal cord injuries: a phase I/II study. Cell Transplant 23 (Suppl 1):S19-S23, 2014

28. Sakuma T, Yamazaki M, Okawa A, Takahashi H, Kato K, Hashimoto M, et al: Neuroprotective therapy using granulocyte colony-stimulating factor for patients with worsening symptoms of compression myelopathy, Part 1: a phase I and IIa clinical trial. Eur Spine J 21:482-489, 2012

29. Sakuma T, Yamazaki M, Okawa A, Takahashi H, Kato K, Hashimoto M, et al: Neuroprotective therapy using granulocyte colony-stimulating factor for patients with worsening symptoms of thoracic myelopathy: a multicenter prospective controlled trial. Spine (Phila Pa 1976) 37:1475-1478, 2012

30. Schäbitz WR, Kollmar R, Schwaninger M, Juettler E, Bardutzky J, Schölzke MN, et al: Neuroprotective effect of granulocyte colony-stimulating factor after focal cerebral ischemia. Stroke 34:745-751, 2003

31. Song S, Kong X, Acosta S, Sava V, Borlongan C, SanchezRamos J: Granulocyte-colony stimulating factor promotes brain repair following traumatic brain injury by recruitment of microglia and increasing neurotrophic factor expression. Restor Neurol Neurosci 34:415-431, 2016

32. Tadie M, d'Arbigny P, Mathé J, Loubert G, Saint-Marc C, Menthonnex P, et al: Acute spinal cord injury: early care and treatment in a multicenter study with gacyclidine. Abstr Soc Neurosci 25:1090, 1999

33. Takahashi H, Yamazaki M, Okawa A, Sakuma T, Kato K, Hashimoto M, et al: Neuroprotective therapy using granulocyte colony-stimulating factor for acute spinal cord injury: a phase I/IIa clinical trial. Eur Spine J 21:2580-2587, 2012
34. Tsai KJ, Tsai YC, Shen CKJ: G-CSF rescues the memory impairment of animal models of Alzheimer's disease. J Exp Med 204:1273-1280, 2007

35. Vaquero J, Zurita M, Rico MA, Bonilla C, Aguayo C, Fernández C, et al: Repeated subarachnoid administrations of autologous mesenchymal stromal cells supported in autologous plasma improve quality of life in patients suffering incomplete spinal cord injury. Cytotherapy 19:349-359, 2017

36. Vaquero J, Zurita M, Rico MA, Bonilla C, Aguayo C, Montilla J, et al: An approach to personalized cell therapy in chronic complete paraplegia: The Puerta de Hierro phase I/II clinical trial. Cytotherapy 18:1025-1036, 2016

37. Varma AK, Das A, Wallace G IV, Barry J, Vertegel AA, Ray SK, et al: Spinal cord injury: a review of current therapy, future treatments, and basic science frontiers. Neurochem Res 38:895-905, 2013

38. Wilson JR, Forgione N, Fehlings MG: Emerging therapies for acute traumatic spinal cord injury. CMAJ 185:485-492, 2013

39. Wu JC, Huang WC, Chen YC, Tu TH, Tsai YA, Huang SF, et al: Acidic fibroblast growth factor for repair of human spinal cord injury: a clinical trial. J Neurosurg Spine 15:216-227, 2011

40. Zhu H, Poon W, Liu Y, Leung GKK, Wong Y, Feng Y, et al: Phase I-II clinical trial assessing safety and efficacy of umbilical cord blood mononuclear cell transplant therapy of chronic complete spinal cord injury. Cell Transplant 25:1925-1943, 2016

\section{Disclosures}

The authors report no conflict of interest concerning the materials or methods used in this study or the findings specified in this paper.

\section{Author Contributions}

Conception and design: Saberi, Derakhshanrad, Joghataei. Acquisition of data: Saberi, Derakhshanrad. Analysis and interpretation of data: Yekaninejad. Drafting the article: Saberi, Derakhshanrad, Joghataei. Critically revising the article: Saberi. Reviewed submitted version of manuscript: all authors. Approved the final version of the manuscript on behalf of all authors: Saberi. Statistical analysis: Yekaninejad. Administrative/technical/material support: Saberi, Joghataei. Study supervision: Saberi.

\section{Correspondence}

Hooshang Saberi: Brain and Spinal cord Injury Research Center (BASIR), Neuroscience Institute, Tehran, Iran. hgsaberi@ yahoo.com. 